\title{
Static Visualization of Temporal Eye-Tracking Data
}

\author{
Kari-Jouko Räihä, Anne Aula, Päivi Majaranta, Harri Rantala, and Kimmo Koivunen \\ Tampere Unit for Computer-Human Interaction, Department of Computer Sciences, \\ FIN-33014 University of Tampere, Finland \\ Tel. +358-3-35518871 \\ \{kjr, aula, curly, hjr, kimmo\}@cs.uta.fi
}

\begin{abstract}
Existing static visualization techniques for eye-tracking data do not make it possible to easily compare temporal information, that is, gaze paths. We review existing techniques and then propose a new technique that treats time as the prime attribute to be visualized. We successfully used the new technique for analysing the visual scanning of web search results listings. Based on our experiences, the new visualization is a valuable tool when the temporal order of visiting Areas of Interest (AOI) is the main focus in the analysis, the AOIs have a natural linear order, there are many AOIs to produce interesting patterns, and the AOIs fill most of the coordinate space being studied.
\end{abstract}

\section{Introduction}

Eye-tracking is increasingly being used to provide insight on a variety of tasks involving visual perception. Eye-trackers provide massive amounts of data that needs to be summarized to make it useful. A variety of numerical metrics, such as fixation duration, number of fixations, and saccade length, are used for this purpose [6].

Before the data can be analyzed statistically with appropriate metrics, it is essential to understand the characteristics of gaze behaviour in the current context. A number of gaze data visualization techniques have been developed for this purpose. They are currently being supported by commercial analysis tools $[4,8]$.

The problem of visualizing temporal gaze data (that is, gaze paths) statically in a compact and usable way still remains a challenge. We first present the existing solutions and then propose a new technique, time plots, that focus on static visualization of the temporal data without cluttering the visualization.

\section{Existing Visualization Techniques}

Most current eye-trackers are based on video technology. They produce coordinates that indicate where the user is looking at using typically a sampling rate from $50 \mathrm{~Hz}$ to $250 \mathrm{~Hz}$. Thus, new coordinates are produced every $20 \mathrm{~ms}$ to every $4 \mathrm{~ms}$, respectively.

A straightforward way of visualizing eye-tracking data is to plot the coordinate stream on top of the observed target. However, this is often problematic for visual analysis of the data: the individual data points carry little information, and in these 
visualizations there are 50 to 250 of them per second. It is a challenging task for the human analyzer to make sense of this stream because of the sheer volume of data. Therefore, the most common way of representing eye-tracking data is to draw a scan path on top of the target image. It consists of fixations, typically shown as circles, and saccades, shown as lines connecting the circles. Figure 1 (a) shows a scan path of a user viewing a web search result listing.
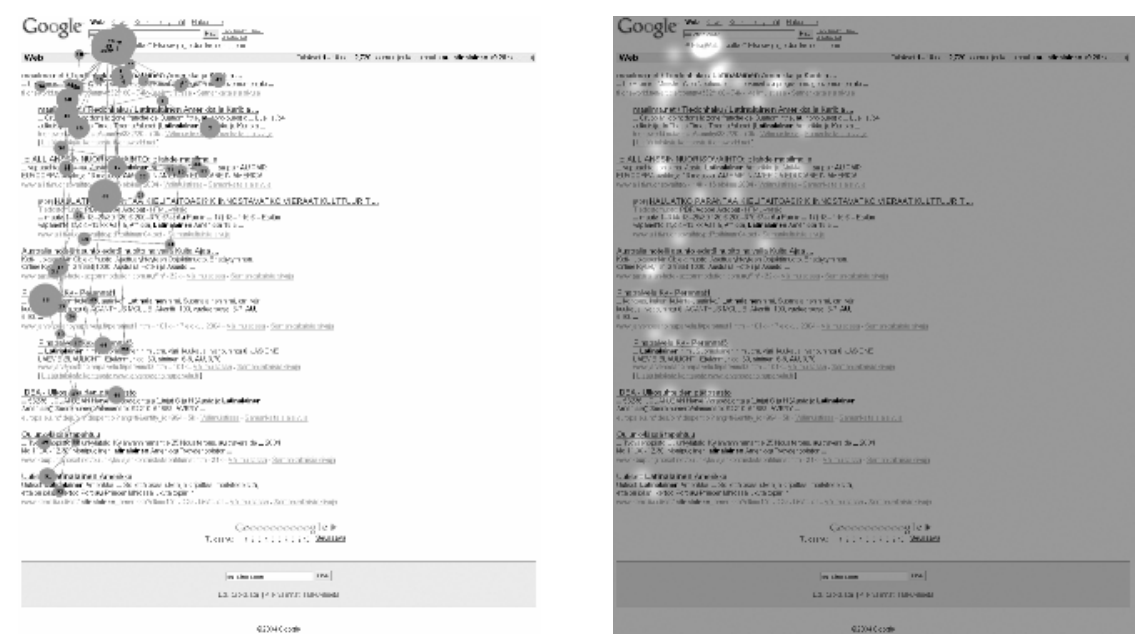

Fig. 1. (a) On the left, a scan path. (b) On the right, the same data visualized as a fixation map.

Scan path visualizations are typically more useful than visualizations of the individual data points, since they group the information into meaningful chunks. However, Figure 1 (a) illustrates the typical problem of gaze path visualizations: the fixations and saccades overlap, making it difficult to figure out the order in which the fixations occurred. The fundamental reason for this problem is that a two-dimensional image is used to represent three-dimensional data: $x$-coordinates, $y$-coordinates, and time. To solve this problem, the third dimension (time) is usually handled in some special way, such as by using colour coding to indicate the order of the saccades. However, as there is no natural ordering of colours for the human observer, the interpretation of the coding is cognitively demanding. Moreover, colour can only give a rough illustration of the order, as these visualizations typically contains tens of saccades.

Another possibility is to collapse the time dimension, so that only the fixations are visualized, not their order. This is a useful way of summarizing numerous gaze paths, even from different users, and it can be effectively used to highlight the points of interest. When such cumulative data is displayed smoothly using fixation maps by David Wooding [9] or its variations, the result is expressive and informative. A sample fixation map is shown in Figure 1 (b). The visualization is less cluttered than the gaze path on the left, but the time dimension has completely disappeared. 


\section{Static Visualization of Temporal Gaze Data}

In some situations the timing information is the crucial aspect in the analysis. Our visualization technique is especially suitable for situations where (1) the exact locations of the fixations are less important than how they land on predefined areas of interest (AOI) in the stimulus; and when (2) the AOIs have a natural linear ordering. This is the case, for instance, when studying gaze behaviour on web pages that are composed of horizontally or vertically arranged blocks of information. Another natural application is studies of menu usage [2, 3].

Prime examples of such web pages are the result listings of web search engines. Figure 2 shows a miniaturized image of such a page, with a gaze path positioned next to it. The data is the same that was used in Figure 1. In the visualization, the $y$ coordinate corresponds to the position in the result listing, and the $x$-coordinate is used to visualize the ordering in time. We call this a time plot of the gaze data. The horizontal location of where the gaze has landed within the area of interest is not shown.

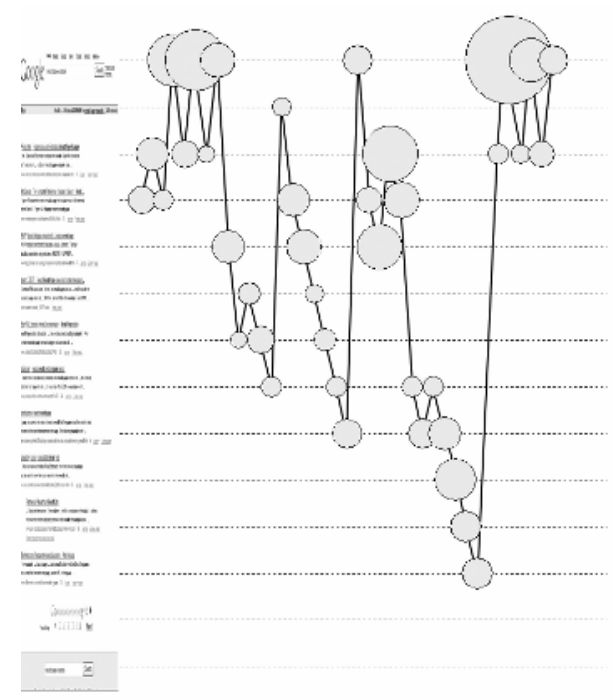

Fig. 2. A time plot of the scan path shown in Figure 1 (a)

Time plot visualizations make it possible to visually observe differences in the gaze behaviour of different users. We have used the technique for analyzing how users perceive search result listings (see [2] for more examples).

The basic time plot approach can be used with variations. For instance, the $y$ coordinate could denote the exact position of the AOI in the coordinate space, and similarly, the $x$-coordinate could denote a relative point in time. In Figure 3 we are visualizing only the order of AOIs, both for the vertical locations $(y)$ and for their visiting order $(x)$. For this analysis task, this solution was found to work well. 


\section{Discussion}

We have proposed a simple new way of visualizing gaze data that facilitates the analysis of gaze paths. We have used it successfully for analyzing the scanning of web search result listings, and believe it to be useful for other visual search tasks as well. Figure 3 illustrates another study where we tried to use this technique. Here the objective was to study the differences of gaze behaviour of designers and consumers while viewing design products. Figure 3 shows that in this case the time plot was less illustrative.

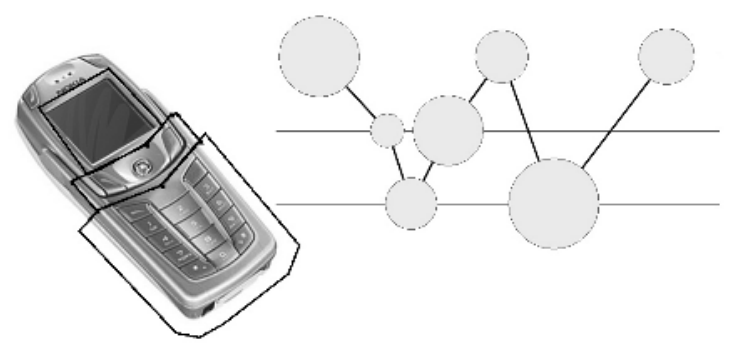

Fig. 3. Time plot (on the right) of a user inspecting the image of a mobile phone. AOIs are highlighted with polygons.

Based on our experiences, we believe the time plot to be a valuable tool when (1) the temporal order of visiting AOIs is the main target of the analysis, (2) the AOIs have a natural linear order, (3) there are sufficiently many AOIs to produce interesting patterns in the time plot, and (4) the AOIs fill most of the coordinate space being studied.

\section{References}

1. Aaltonen, A., Hyrskykari, A., and Räihä, K.-J.: 101 spots, or how do users read menus? In Proc. CHI 1998, ACM Press (1998) 132-139

2. Aula, A., Majaranta, P., and Räihä, K-J.: Eye-tracking reveals the personal styles for search result evaluation. In Proc. INTERACT 2005, Rome, September 2005

3. Byrne, M.D., Anderson, J.R., Douglass, S., and Matessa, M.: Eye tracking the visual search of click-down menus. In Proc. CHI 1999, ACM Press (1999) 402-409

4. Eye Response Technologies: GazeTracker. Available at http://www.eyeresponse.com/

5. Feusner, M.: Eye-tracking visualizations. Available at http://www.hci.cornell.edu/eyetracking/visualizations.php

6. Jacob, R.J.K., and Karn, K.S.: Eye tracking in human-computer interaction and usability research: Ready to deliver the promises (section commentary). In The Mind's Eye: Cognitive and Applied Aspects of Eye Movement Research, ed. by J. Hyönä, R. Radach, and H. Deubel, pp. 573-605, Amsterdam, Elsevier Science, 2003

7. Outing, S., and Ruel, L.: The Best of Eyetrack III: What We Saw When We Looked Through Their Eyes. Available at http://www.poynterextra.org/eyetrack2004

8. Tobii Technology: ClearView. Available at http://www.tobii.com/

9. Wooding, D.: Fixation maps: quantifying eye-movement traces. In Proc. ETRA'02, ACM Press (2002), 31-36 